\title{
L'émergence de la notion de classique dans la musique chez Amadeus Wendt (1783-1836)
}

Zur Entstehung des Begriffs der Klassik in der Musik bei Amadeus Wendt About the rise of a concept "musical classicism" in Amadeus Wendt's work

\section{Alexandre Chèvremont}

\section{OpenEdition}

\section{Journals}

Édition électronique

URL : http://journals.openedition.org/ceg/5651

DOI : $10.4000 /$ ceg. 5651

ISSN : 2605-8359

Éditeur

Presses Universitaires de Provence

Édition imprimée

Date de publication : 1 octobre 2013

Pagination : $59-72$

ISSN : 0751-4239

Référence électronique

Alexandre Chèvremont, «L'émergence de la notion de classique dans la musique chez Amadeus Wendt (1783-1836) », Cahiers d'Etudes Germaniques [En ligne], 65 | 2013, mis en ligne le 01 octobre 2019, consulté le 25 novembre 2020. URL : http://journals.openedition.org/ceg/5651 ; DOI : https:// doi.org/10.4000/ceg.5651 


\title{
L'émergence de la notion de classique dans la musique chez Amadeus Wendt (1783-1836)
}

\author{
Alexandre CHÈVREMONT
}

Lyon

Amadeus Wendt est le premier à avoir parlé d'une «période dite classique » (sogenannte classische Periode) à propos de la musique ${ }^{1}$. Dans son dernier ouvrage Ueber den gegenwärtigen Zustand der Musik besonders in Deutschland, und wie er geworden (De l'état actuel de la musique, particulièrement en Allemagne, et de son origine), il canonise Haydn, Mozart et Beethoven comme les trois grands maîtres de ce qu'on appelle, à sa suite, le « classicisme viennois », sans aucune référence à l'Antiquité. Wendt veut dire qu'ils ont ouvert à l'art musical des possibilités nouvelles qui lui ont permis de parvenir à son sommet, mais aussi qu'ils représentent un équilibre qui s'est ensuite perdu. Nous proposons de traduire die Klassik par «le classique » plutôt que par «le classicisme », malgré le germanisme, pour rester au plus près du sens véritable du concept avancé par Wendt. Wendt est souvent considéré comme un professeur de philosophie de Leipzig, amateur très éclairé de musique, mais ne dépassant pas le niveau d'un épigone de Hegel $^{2}$. Cependant, puisqu'il présente l'application du classique à la musique

${ }^{1}$ Amadeus WENDT, Ueber den gegenwärtigen Zustand der Musik besonders in Deutschland, und wie er geworden, Göttingen, Dieterich, 1836, p. 3 : « Es ist aber unmöglich von der musikalischen Gegenwart zu sprechen, ohne auf die sogenannte classische Periode und die Coryphäen zurückzugehen, durch welche sie vorbereitet worden ist. Hier leuchtet uns das Kleeblatt : Haydn, Mozart, Beethoven entgegen " (il est impossible de parler de l'actualité musicale sans revenir à la période appelée classique et aux coryphées qui l'ont préparée ; ici, c'est la triade : Haydn, Mozart, Beethoven qui nous illumine). Dans son essai Zum Begriff der Klassik in der Musik (Sur le concept du classique dans la musique), in Deutsches Jahrbuch der Musikwissenschaft, XI, 1966, Ludwig FINSCHER fait de Wendt l'inventeur du concept de classique en musique.

2 Tous les auteurs traitant de Wendt (Bernadette Collenberg-Plotnikov, Hans Heinrich Eggebrecht, Ludwig Finscher, Edward Lippman, Alain Patrick Olivier, Robin Wallace pour n'en citer que quelques-uns) insistent sur son hégélianisme latent ou affiché. Le lien avec Hegel est présenté soit comme une communauté de pensée, soit carrément comme une sujétion servile. Hegel est une figure assurément écrasante et personne ne songe à lui comparer Amadeus Wendt. Mais faut-il en conclure que Wendt n'a rien d'original à nous dire sur le sujet ? 
comme étant déjà en usage, on peut estimer que ce n'est pas Hegel, mais les romantiques, tels que Ludwig Tieck, Wilhelm Heinrich Wackenroder et surtout E. T. A. Hoffmann, qui lui ont préparé le terrain et ont déjà pensé la chose. Ce sont eux qui se sont attachés avant lui à montrer que la musique de leur temps était parvenue, ou allait parvenir, à son plein épanouissement.

Dans leur approche, la musique est un art ayant enfin trouvé son autonomie et la possibilité d'exprimer ce qu'il a de plus propre, à travers l'essor de la musique instrumentale. Dès 1799, Ludwig Tieck, tout en reconnaissant que la musique n'a pas encore connu sa période classique, l'encourage à suivre son propre chemin en explorant les possibilités symphoniques qui s'offrent désormais à elle ${ }^{3}$. Hoffmann, quant à lui, considère dans son célèbre compte rendu de la cinquième symphonie de Beethoven, datant de 1810, que "lorsqu'on parle de la musique comme d'un art autonome, on ne devrait jamais penser qu'à la musique instrumentale ${ }^{4}$ ». Dans sa symphonie, Beethoven serait allé encore plus loin sur la voie tracée par Haydn et Mozart, qui mène à l'autonomie complète de la musique instrumentale et lui permet ainsi d'atteindre son sommet, c'est-à-dire ce que Wendt appelle un quart de siècle plus tard sa "période classique». Aussi bien Hoffmann que Tieck et Wackenroder ne songent qu'à la musique instrumentale. La musique vocale est selon eux un genre hybride où la musique, mêlée à la poésie, et par là esclave d'une parole et d'une action, ne peut trouver le chemin d'une totale liberté. Il faut briser les chaînes qui empêchent la musique d'atteindre sa propre essence. La symphonie beethovénienne est venue comme par miracle accomplir le programme romantique qui l'avait précédée. Elle est ainsi devenue le modèle de l'autonomie de la musique.

Un examen plus attentif oblige à interroger ce miracle avec davantage de circonspection et à se demander si la musique instrumentale est vraiment seule concernée dans cette affaire. Après tout, Hoffmann lui-même appelait le Don Giovanni de Mozart «l'opéra de tous les opéras ${ }^{5}$ » : celui qui a ouvert la route aux compositions titanesques de Beethoven dans le registre de la symphonie a atteint des sommets dans un genre de la musique vocale. Et de

\footnotetext{
3 Voir Ludwig TIECK et Wilhelm Heinrich WACKENRODER, Épanchements d'un moine ami des arts, suivi de Fantaisies sur l'art, traduction de l'allemand, introduction et notes par Charles Le Blanc et Olivier Schefer, Paris, José Corti (Domaine romantique), 2009, p. 254-262.

${ }^{4}$ E. T. A. Hoffmann, Schriften zur Musik, München, Winkler, 1963, p. 34 : «Wenn von der Musik als einer selbstständigen Kunst die Rede ist, sollte immer nur die Instrumentalmusik gemeint sein. ». Traduit par Alain MontANDON, in E. T. A. Hoffmann, Écrits sur la musique, Lausanne, L'Âge d'homme, 1985, p. 38.

${ }^{5}$ Recension du mercredi 20 septembre 1815 de Don Juan, opéra en deux parties de Mozart, in ibid., p. 297 : « Nur in der großen Schwürigkeit dieser Oper aller Opern, von deren poetischer Tiefe auch wohl mancher Zuschauer nur hin und her was ahnen mag, liegt es, daß bis jetzt die Darstellung nicht die zu wünschende Ründe erhalten hat. ") Traduction d'Alain MONTANDON, p. 233 : « La grande difficulté de cet opéra de tous les opéras, dont la profondeur poétique n’est pressentie que çà et là par certains spectateurs, fit que, jusqu'à présent, sa représentation n'a pas reçu la perfection souhaitable. »
} 
toute évidence, Mozart est connu dans ce premier tiers du XIX ${ }^{\mathrm{e}}$ siècle au moins autant et sans doute davantage pour ses opéras que pour sa musique de chambre ou ses symphonies. Alors, Mozart aura été un maître, peut-être, de la musique instrumentale, mais aussi de son emploi dans le domaine de la musique vocale, tout au moins du genre de l'opéra, en laissant de côté la question très épineuse du rôle de l'instrumentation dans la musique vocale sacrée. Amadeus Wendt a écrit une recension du Fidelio de Beethoven, qu'il ne considère assurément pas comme inférieure à ses compositions instrumentales, même si cette œuvre fait selon lui exception à l'esprit général de la musique de Beethoven ${ }^{6}$. L'inventeur de la notion de classique dans la musique s'est d'abord intéressé à l'unique opéra de Beethoven, le compositeur que Hoffmann considérait comme le parangon de la musique instrumentale romantique.

Les trois maîtres que Hoffmann appelle des romantiques sont considérés par Wendt comme les hérauts de la période classique. Certes, derrière la différence des termes, l'idée semble être la même : la musique, romantique par nature, accomplit son essence dans sa période classique, qui fournit le modèle de ce qu'elle peut et doit être. Jean-François Candoni fait remarquer avec raison dans un ouvrage récent qu' ' on peut légitimement se demander si ce paradoxe d'une musique d'essence romantique qui s'incarne dans une esthétique classique n'est pas présent également sous une forme latente dans l'œuvre de Hoffmann ${ }^{7}$ ». Mais la différence des mots est-elle si anodine en l'occurrence ? Dans ce glissement terminologique, il y a changement d'accent, déplacement d'un centre de gravité. Les romantiques ont promu l'autonomie de la musique instrumentale, mais l'émergence de la notion de musique classique, n'est-ce pas aussi, et peut-être même avant tout, la pensée et l'esthétique d'une musique d'opéra?

\section{Du romantisme instrumental chez Hoffmann}

Le parti pris de Hoffmann, dans le compte-rendu de la cinquième symphonie de Beethoven, semble exclusivement favorable à la musique instrumentale. Il couplera ce texte à celui sur les trios op. 70 du même compositeur, écrivant ainsi l'un des textes les plus fameux du romantisme sur la musique, La musique instrumentale de Beethoven (Beethovens Instrumentalmusik), dans la deuxième série des Kreisleriana. Hoffmann

\footnotetext{
6 Amadeus WENDT, « Gedanken über die neuere Tonkunst, und van Beethovens Musik, namentlich dessen Fidelio », Allgemeine musikalische Zeitung, vol. 17, $\mathrm{n}^{\circ}$ 21, 24 mai 1815, p. 345-353. Wendt et Hoffmann furent tous deux des rédacteurs d'importance de cette prestigieuse revue musicale. Voir Stefan KUNZE, Ludwig van Beethoven, Die Werke im Spiegel seiner Zeit - Gesammelte Konzertberichte und Rezensionen bis 1830, Laaber, Laaber Verlag, 1996, p. 173-204.

${ }^{7}$ Jean-François CANDONI, Penser la musique au siècle du romantisme - Discours esthétiques dans l'Allemagne et l'Autriche du XIX siècle, Paris, PUPS, 2012, p. 64.
} 
s'inscrit de la sorte dans l'héritage de la promotion romantique de la musique instrumentale, telle qu'elle se fait jour déjà dans les Fantaisies sur l'art de Tieck et de Wackenroder, où c'est une musique de scène de Johann Friedrich Reichardt sur la tragédie de Macbeth qui sert d'exemple à ce que les auteurs souhaitent en matière de musique. Était-ce faute de mieux ? En tout cas, avec Beethoven, le romantisme instrumental a enfin trouvé le champion qu'il appelait de ses vœux. L'esthétique romantique a précédé la musique qui l'incarne. Le programme est venu avant l'œuvre.

Pour Hoffmann, « la musique ouvre à l'homme un royaume inconnu totalement étranger au monde sensible qui l'entoure, et où il se dépouille de tous les sentiments qu'on peut nommer pour plonger dans l'indicible ${ }^{8} »$. Elle s'oppose diamétralement aux arts plastiques et à la poésie, art de la parole qui nous montrent quelque chose de tangible et donc de terrestre. Tant que nous pouvons voir ou comprendre, nous sommes limités par notre vue et notre entendement. L'esprit de la sculpture, que la Grèce antique a portée à sa perfection, reste «tendu vers l'incarnation sensuelle ${ }^{9} »$. C'est le christianisme et sa pure spiritualité qui ont permis à l'essence véritable de la musique de se développer et de s'émanciper, selon l'article de 1814 intitulé «Alte und neue Kirchenmusik» (Musique sacrée ancienne et moderne). La musique vocale sacrée qui s'est développée au Moyen Âge et à la Renaissance est indifférente au texte, qu'elle n'accompagne pas : seule compte la polyphonie des voix et le chant a une valeur propre, quelles que soient les paroles chantées. Ainsi la musique se libère des attaches terrestres et nous ouvre sur son propre monde. Comment la musique pourrait-elle, selon cette vision des choses, ne pas être encombrée par la représentation visible, et la parole, bien plus précise qu'elle, que l'opéra l'oblige à accompagner? Si elle veut parvenir à son sommet, elle doit se libérer de ces entraves :

\footnotetext{
Beethoven est un compositeur purement romantique, et donc authentiquement musical. C'est peut-être pour cela qu'il est moins à l'aise dans la musique vocale, qui ne laisse aucune place au désir sans objet, mais se contente de transposer dans le domaine de l'infini les émotions exprimées par les mots, et c'est sans doute aussi pour cette raison que sa musique instrumentale ne trouve que rarement un écho dans le public.
}

${ }^{8}$ Recension de la cinquième symphonie de Beethoven, in HofFMANN, Schriften zur Musik, p. 34 : «Die Musik schließt dem Menschen ein unbekanntes Reich auf; eine Welt, die nichts gemeint hat mit der äußern Sinnenwelt, die ihn umgibt, und in der er alle durch bestimmbaren Gefühle zurückläßt, um sich dem Unaussprechlichen hinzugeben ». Traduction d'Alain MONTANDON, p. 38.

${ }^{9}$ Ibid., p. 212 : «Eben dieses ihres eigentümlichen Wesens halber konnte die Musik nicht das Eigentum des antiken Welt, wo alles auf sinnliche Verleiblichung ausging, sein, sondern mußte dem modernen Zeitalter angehören. » Traduction d'Alain MONTANDON, p. 175: « Cette essence spécifique [de la musique] interdisait au monde antique, tout entier tendu vers l'incarnation sensuelle, de posséder la musique ; celle-ci ne pouvait appartenir qu'aux temps modernes. »

${ }^{10}$ Ibid., p. 36 : «Beethoven ist ein rein romantischer (eben deshalb ein wahrhaft musikalischer) Komponist, und daher mag es kommen, daß ihm Vokalmusik, die unbestimmtes 
Tout ceci semble faire de la musique d'opéra un genre inférieur à la musique instrumentale. Car la musique y accompagne un texte et doit se plier au déroulement d'une action scénique. Hoffmann loue Gluck d'avoir su comprendre que l'opéra est une totalité, et dans l'essai «Sur un jugement de Sacchini et sur ce qu'on appelle "l'effet" dans la musique », inclus dans les Kreisleriana, il rappelle que contrairement à lui « les Italiens ne se sont pas élevés jusqu'à la perspective qui permet de voir ce que doit être l'opéra, un tout englobant le mot, la musique et l'action ${ }^{11} »$. Mais cet éloge de Gluck a son revers : comment la musique peut-elle y trouver la liberté que laisse la symphonie à l'imagination débordante du compositeur? D'une part, le symphoniste moderne dispose de moyens plus riches que ceux de la polyphonie vocale sacrée et, d'autre part, il n'est pas limité par les bornes de l'opéra. Il faut en conclure que Hoffmann se refuse à laisser la place dans sa conception romantique de la musique à cette harmonieuse totalité d'une musique, d'une parole et d'une action qu'est l'opéra, ou du moins qu'il n'en fait pas un modèle. Le parangon de la musique romantique, c'est la musique instrumentale, et la musique d'opéra n'y est tolérée que dans la mesure où elle parvient malgré ses propres obstacles à se rapprocher d'elle.

\section{Hoffmann et l'opéra}

Les recensions ne manquent pas où Hoffmann s'intéresse à l'opéra. Luimême compositeur, il exècre Rossini, mais admire Gluck, Mozart et Spontini.

Sehnen nicht zuläßt, sondern nur die durch Worte bezeichneten Affekte, als in dem Reich des Unendlichen empfunden, darstellt, weniger gelingt und seine Instrumentalmusik selten die Menge anspricht. » Traduction d'Alain MONTANDON, p. 40.

11 E. T. A. Hoffmann, Poetische Werke in sechs Bänden. Mit einer Einleitung von Hand Mayer, Berlin, Aufbau, 1963, vol. 1, p. 448 : « Die Italiener erhoben sich nicht zu der Ansicht, daß die Oper in Wort, Handlung und Musik als ein Ganzes erscheinen, und dieses untrennbare Ganze im Totaleindruck auf den Zuhörer wirken müsse ; die Musik war ihnen vielmehr zufällige Begleiterin des Schauspiels und durfte nur hin und wieder als selbständige Kunst, und dann für sich allein wirkend, hervortreten. So kam es, daß im eigentlichen Fortschreiten der Handlung alle Musik flach und unbedeutend gehalten wurde, und nur die Prima Donna und der Primo Huomo in ihren sogenannten Szenen in bedeutender oder vielmehr wahrer Musik hervortreten durften. Hier galt es aber dann ohne Rücksicht auf den Moment der Handlung nur den Gesang, ja oft auch nur die Kunstfertigkeit der Sänger im höchsten Glanze zu zeigen. » (Les Italiens ne se sont pas élevés jusqu'à la perspective qui permet de voir ce que doit être l'opéra, un tout englobant le mot, la musique et l'action, qui inséparablement produisent une impression globale sur l'auditeur ; pour eux, la musique accompagne ici ou là le spectacle, et lorsqu'enfin on l'autorise à passer au premier plan, c'est seulement comme un art autonome, qui n'a d'effet que par soi-même. Le progrès proprement dit de l'action n'est donc soutenu que par une musique plate et insignifiante, et c'est seulement dans leurs soi-disant scènes que la prima donna et le primo huomo [sic] nous laissent le droit d'entendre de la vraie musique, qui a quelque chose à dire. Mais même alors le chant reste indifférent à la situation, et n'est souvent là que pour faire étalage de la technique brillante du chanteur.) Il est notable que Hoffmann reproche à la musique de l'opéra italien de rester autonome (selbständig) alors que c'est précisément ce qu'il loue dans le romantisme instrumental de Beethoven. 
Il sent souffler dans Don Giovanni le même esprit romantique que celui qui anime la symphonie ou la musique de chambre de Beethoven, dans la mesure où malgré son souci nécessaire de la scène, la musique y garde son pouvoir mystérieux, son autonomie transcendante. La musique est rabaissée par le livret lorsqu'il lui coupe les ailes parce que le librettiste a produit un texte sans poésie. Mais elle le sublime si celui-ci se prête à son essor. La musique, même si son éclat vient d'un autre monde, peut et doit éclairer le nôtre de façon à rendre l'opéra encore plus poétique. Dans son compte-rendu de L'Orphelinat de Weigl, Hoffmann avance que le texte doit laisser libre l'imagination du compositeur pour qu'il saisisse l'idée qui a inspiré le librettiste sans chercher à suivre mot à mot le déroulement de la scène. Il y a donc une idée poétique qui est commune au texte et à la musique, même si celle-ci doit s'affranchir du sens strict des mots pour parvenir à elle. En réalité, l'opéra fait donc partie intégrante du romantisme :

\begin{abstract}
Il est absolument condamnable de choisir comme sujet d'opéra des scènes de la vie de tous les jours, qui sont pour ainsi dire à l'opposé de tout romantisme, car l'opéra élève la nature humaine à une puissance supérieure : le langage y est chant, chaque expression de la passion est suivie d'accords harmonieux, comme le sont les paroles des fées dans le conte célèbre, bref, l'opéra n'existe que dans le royaume enchanté du romantisme. ${ }^{12}$
\end{abstract}

L'illusion théâtrale permet en outre à l'opéra de nous transporter dans un monde fictif et nous évite tout rappel de la réalité, comme l'explique le texte du Parfait machiniste (Der vollkommene Maschinist) publié dans la première série des Kreisleriana. La représentation scénique du visible débouche sur l'invisible, le sensible devient purement imaginaire et la musique favorise ce processus hallucinatoire. La preuve que l'opéra se prête à l'autonomie de la musique, c'est que ce spectacle enchanté nous transporte dans le monde de l'ailleurs que nous ouvre l'art. Même à l'audition de la Bataille de Vitoria de Beethoven, Hoffmann relève une réaction analogue qui sauve de sa médiocrité cette symphonie de bataille descriptive ${ }^{13}$. Il est ainsi permis d'aller

${ }^{12}$ HoffmanN, Schriften zur Musik, p. 53 : «Vollends als Gegenstand der Oper, die die menschliche Natur höher potenziert, wo die Sprache Gesang ist, und, wie in jenem Märchen den Reden der Fee, jedem Akkorde der Leidenschaft Akkorde nachklingen, und die überhaupt nur in dem wundervollen Reiche der Romantik existiert, ist es gewiss verwerflich, Szenen der gemeinen Lebens zu wählen, die jeder Romantik geradezu entgegenstreben. » Traduction d'Alain MONTANDON, p. 52.

${ }^{13}$ Ibid., p. 335 : « Schreiber dieses bemerkte mit wahrer Lust einen Militär von höherm Range, der ganz in sich verloren dastand, und als nun Schlag auf Schlag der Sturm der Dissonanzen den höchsten Punkt, die Krisis der Schlacht bezeichnete, unwillkürlich nach dem Säbel griff, als wolle er sich an die Spitze des Bataillons setzen zum entscheidenden Angriff. Ein mildes Lächeln überflog sein Gesicht, als er in dem Augenblick die Täuschung des innern Sinns gewahrte. » Traduction d'Alain MONTANDON, p. 259: "L'auteur de ces lignes a remarqué, avec un vif plaisir, un officier supérieur qui se trouvait là, tout à fait recueilli, et qui, lorsque, coup sur coup, l'assaut des dissonances symbolisa le point culminant, la crise décisive de la bataille, saisit machinalement son sabre, comme s'il voulait se porter à la tête de son bataillon pour livrer l'attaque déterminante. Il remarqua aussitôt son hallucination, et un sourire 
jusqu'à la conclusion très paradoxale de Peter Schnaus, qui considère l'opposition de la musique instrumentale et de la musique vocale comme secondaire dans la conception de la musique du romantisme hoffmannien :

\begin{abstract}
Selon Hoffmann, il faut attribuer à la musique vocale, pour autant qu'elle soit aussi de la musique, le même " esprit romantique » qu'à la musique instrumentale. [...] Et pour le dire de manière paradoxale, la musique instrumentale interprétée comme romantique apparaît comme une espèce de musique vocale sans mots, c'est-à-dire au-delà des mots, de même que la musique vocale contient toujours déjà en elle la tendance romantique de la musique instrumentale.

Si l'on suit cette argumentation, la musique vocale et la musique instrumentale perdent dans la conception hoffmannienne de la musique leur antagonisme et deviennent presque des aspects simplement différents d'une seule et même chose. $^{14}$
\end{abstract}

La promotion de la musique instrumentale comme art romantique par excellence n'exclut donc pas la musique vocale, qui est aussi de la musique. Le romantisme exacerbé de Beethoven ne parvient certes pas à se satisfaire des limites de la parole et de l'action scénique. Mais c'est le génie de Mozart d'avoir su adapter les progrès de la musique instrumentale, dont il est un des compositeurs les plus avancés, aux exigences propres de l'opéra. Dans Musique sacrée ancienne et moderne, Hoffmann analyse le déclin de la musique religieuse de son époque et lui oppose en bloc les progrès de la musique profane, qu'elle soit instrumentale ou vocale. L'opposition ne passe pas entre opéra et symphonie, mais entre l'ancienne musique, pénétrée de religiosité, et la nouvelle, qui ne peut trouver sa place qu'au concert ou au théâtre et qui n'arrive à sa perfection et à sa légitimité que dans le monde du romantisme. Certes, les textes ne manquent pas où Hoffmann dénigre la musique de Rossini, jugée légère et inconsistante. Mais ce n'est pas parce que c'est de la musique d'opéra. On trouve les mêmes reproches envers des symphonies de bataille inféodées à l'imitation de scènes extérieures. C'est le traitement génial de la musique qui importe plus que le genre pratiqué. En définitive, Hoffmann est loin d'exclure l'opéra du romantisme, mais il n'en fait pas non plus un modèle. L'opéra ne devient romantique que si la musique y est aussi libre de déployer sa puissance propre que dans la musique instrumentale. Cela n'est possible qu'au compositeur qui saisit l'idée

fugitif et léger passa sur son visage ». Hoffmann par ailleurs ne cache pas son aversion pour les symphonies de bataille, notamment au début de la recension de la cinquième symphonie.

Peter Schnaus, E. T. A. Hoffmann als Beethoven-Rezensent der Allgemeinen musikalischen Zeitung, München-Salzburg, Emil Katzblicher, 1977, p. 133 : «Im Sinne Hoffmanns muß also der Vokalmusik, eben insofern sie auch Musik ist, der „gleiche romantische Geist“" zugeschrieben werden wie der Instrumentalmusik. [...] Und die romantisch gedeutete Instrumentalmusik erscheint, paradox formuliert, als eine Art Vokalmusik ohne Worte bzw. jenseits der Worte, so wie die Vokalmusik die romantische Tendenz der Instrumentalmusik immer schon in sich enthält. Folgt man dieser Argumentation, so verlieren Vokalmusik und Instrumentalmusik in Bezug auf Hoffmanns Musikanschauung ihren gegensätzlichen Charakter, werden fast zu bloßen Aspekten ein und derselben Sache. » 
métaphysique, l'esprit du livret au-delà des mots et des scènes visibles. La musique reste l'âme dont la représentation et la parole ne sont qu'une enveloppe extérieure :

Toute passion - amour, haine, fureur, désespoir - représentée à l'opéra, la musique la revêt de l'éclat d'une pourpre romantique, et même les sentiments que nous éprouvons dans la vie nous la font quitter pour le royaume de l'infini. Tel est le pouvoir magique de la musique. ${ }^{15}$

\section{Wendt et le classique}

Amadeus Wendt s'accorde avec Hoffmann pour canoniser Haydn, Mozart et Beethoven comme les trois maîtres ayant permis à la musique d'atteindre le sommet de sa perfection. Sur ce point, l'accord est total entre eux. Mais Hoffmann considère cette triade comme un approfondissement vers la vérité du romantisme, dont Beethoven représente le sommet. Le compte-rendu de sa cinquième symphonie le place pour cette raison au-dessus de tout. Wendt au contraire, dans De l'état actuel de la musique, pense que "ce qui fait de Mozart le point d'équilibre de la période classique, c'est le mariage suprêmement beau du chant et de la musique instrumentale en un sens authentiquement allemand. Le sommet de sa création est le plus grand des opéras allemands : Don Juan ${ }^{16}{ }$. Hoffmann et Wendt admirent tous deux les trois maîtres et les mêmes œuvres. Mais le centre de gravité s'est déplacé. Hoffmann cherche en eux le romantisme, la nostalgie d'un monde transcendant, l'expression de l'indicible. Wendt y trouve l'expression du classique, c'est-à-dire non seulement le sommet et par conséquent le modèle de la musique en général, mais aussi le souci de l'équilibre et le respect de certaines limites.

Elisabeth Eleonore Bauer estime que Wendt place Mozart au sommet de la triade classique à cause de l'équilibre parfait dans ses symphonies entre forme et contenu, entre fantaisie et réflexion, contrairement à celles de Beethoven où l'abondance de la matière l'emporte sur la forme et déstructure l'organisation du tout ${ }^{17}$. Mais c'est, plus encore que la symphonie, l'opéra mozartien qui est au sommet de la période classique, en raison de sa faculté à mêler l'art du chant à la masse instrumentale. Un peu plus loin dans son ouvrage, Wendt nuance son éloge de Beethoven, maître incontesté de la

\footnotetext{
${ }^{15}$ HofFMAnN, Schriften zur Musik, p. 34-35 : « Jede Leidenschaft - Liebe - Haß - Zorn Verzweiflung etc. wie die Oper sie uns gibt, kleidet die Musik in den Purpurschimmer der Romantik, und selbst das im Leben Empfundene führt uns hinaus aus dem Leben in das Reich des Unendlichen. So stark ist der Zauber der Musik. ». Traduction d'Alain MonTANDON, p. 39.

${ }^{16}$ WENDT, Zustand, p. 5-6 : « Was aber Mozart zum Mittelpunkte der classischen Periode macht, das ist die schönste Vermählung von Gesang und Instrumentalmusik in ächt deutschem Sinne. Der Gipfel seiner Schöpfung ist die größte deutsche Oper : Don Juan. »

17 Elisabeth Eleonore BAUER, Wie Beethoven auf den Sockel kam - die Entstehung eines musikalischen Mythos, Stuttgart / Weimar, Metzler, 1992 (limited preview 1987), p. 317.
} 
musique instrumentale : «Beethoven, dans cet esprit, s'est rendu maitre de l'esprit de chaque instrument. Son œuvre a donné à la musique instrumentale toute sa puissance et, ainsi, à la musique l'autonomie que l'art réclame. Mais le chant lui est toujours d'une certaine manière demeuré étranger ${ }^{18} »$. Le héros de la musique instrumentale a son talon d'Achille. Son allure colossale lui interdit la grâce du chant.

La lecture de l'ouvrage de Wendt qui prétend englober toute l'histoire de l'art, Des grandes périodes des beaux-arts, ou de l'art au cours de l'histoire universelle $^{19}$, confirme à tous égards ce point de vue. Wendt y insiste sur les dangers que court l'art du chant depuis que Beethoven a donné son plein essor à la musique instrumentale et que celle-ci s'est également imposée dans la fosse d'orchestre de l'opéra. Il cherche un remède à cet essor dans les vertus propres de la vocalité, montrant ainsi la nécessité de retrouver la synthèse du chant et de l'orchestre que Mozart avait si bien su incarner :

\begin{abstract}
Depuis Mozart et Winter, dans la plupart des opéras allemands, les modulations trop fréquentes ont desséché la mélodie et lui ont fait perdre de son naturel. En France - mais aussi en Allemagne, où les opéras français de Cherubini, de Spontini, etc. ont comblé le manque d'œuvres nouvelles -, on avait le plus grand besoin de renouveler le chant mélodique. C'est l'Italien Gioachimo <sic $>$ Rossini (né en 1792) qui est venu le satisfaire. Ses mélodies fraîches, plaisantes et caressantes, influencées par Paer, ont cependant une harmonie plus riche et des effets instrumentaux modernes ; Rossini a su ainsi relever la grâce de l'art du chant italien. De telles qualités ont fait de lui le dieu chéri du grand public partout dans le monde. ${ }^{20}$
\end{abstract}

Rossini, que Hoffmann exécrait, présenté comme un remède aux excès de la musique instrumentale héritière de Beethoven! Wendt a traduit en allemand et refondu la Vie de Rossini ${ }^{21}$. Stendhal était sensible au pur chant

${ }^{18}$ WENDT, Zustand, p. 7 : «Mit diesem Geiste bemächtigte sich Beethoven der Geister aller Instrumente, und Herrschaft der Instrumentalmusik, damit aber Erhebung der Musik zu ihrer kunstmässiger Selbständigkeit war das Resultat seines Schaffens. Der Gesang aber blieb ihm auf gewisse Weise immer fern. »

19 Amadeus WENDT, Ueber die Hauptperioden der schönen Kunst, oder die Kunst im Laufe der Weltgeschichte, Leipzig, Johann Ambrosius Barth, 1831.

${ }^{20}$ Ibid., p. 310-311: «In den meisten deutschen Opern seit Mozart und Winter sah man die Melodie, durch Harmoniewechsel bestimmt, häufig trocken und unnatürlich werden. In Frankreich, so wie in Deutschland, wo man auch den Mangel neuer Werke durch die französischen Opern Cherubini's, Spontini's u. ausgefüllt hatte, war ein Bedürfniß nach neuer Gesangsmelodie in der Masse vorhanden. Diesem Bedürfnisse kam der Italiener Gioachimo Rossini (geb. 1792) entgegen, der, sich zuerst an Paer anschließend, den Reiz des italienischen Kunstgesanges durch eine Fülle frischer, höchstwohlgefâlliger und schmeichelnder Melodieen und durch die Umgebung reicherer Harmonie und neuer Instrumentaleffecte zu steigern wußte. Solche Eigenschaften machten ihn zum vergötterten Lieblinge des großen Publicums aller Welttheile. »

${ }^{21}$ Amadeus WENDT, Rossini's Leben und Treiben, vornehmlich nach den Nachrichten des Herrn v. Stendhal geschildert und mit Urtheilen der Zeitgenossen ûber seinen musikalischen Charakter begleitet von Amadeus Wendt, Hildesheim / Zürich / New York, Georg Olms, 2003 $\left(1824^{1}\right)$. Il faut souligner, au sujet de Rossini, qu'il passait à son époque pour un compositeur n'ayant aucun souci du texte ni de l'action. Soit cela lui était reproché, comme chez Hoffmann, 
italien dont Rossini représente pour lui à la fois l'apogée et le terme. Wendt loue le cygne de Pesaro pour ces qualités héritées de la tradition italienne, mais il lui rend également hommage pour avoir su intégrer à ses compositions, et notamment à son dernier opéra, Guillaume Tell, les progrès les plus récents du style instrumental. Il incarne de ce fait un nouvel équilibre entre le chant et l'orchestre, équilibre que Beethoven et ses héritiers dans le domaine de l'opéra ont rompu. Dans sa recension de Fidelio en 1815, Wendt insistait déjà sur l'ambivalence de tout progrès :

Certains tiennent le développement artificiel de la musique instrumentale pour une corruption de l'art, ce qui n'est pas dépourvu de fondement, dans la mesure où le plus haut degré de développement d'une chose contient en même temps le germe de son déclin. 22

Après le sommet beethovénien, il faut trouver les solutions à la décadence d'un art qui a trop progressé. L'opéra est le seul lieu où la musique instrumentale peut espérer retrouver un équilibre qui lui vienne des limites imposées par la grâce naturelle du chant.

\title{
La musique instrumentale à l'opéra
}

Dès le début de son compte-rendu du Fidelio de Beethoven en 1815, Amadeus Wendt s'en prend aux dégénérescences de la musique instrumentale qui, en raison de l'autonomie qu'elle a gagnée par rapport à l'art du chant, présente le risque de devenir un jeu vide de sons ne voulant rien dire :

\begin{abstract}
La musique exerce pleinement son invisible puissance dans la musique instrumentale, où elle est souveraine. C'est ici pourtant qu'elle dégénère le plus facilement, pour ceux qui ne savent pas comment contrôler les esprits des sons avec la baguette magique du génie, et qui jouent artificiellement avec les sons. Il est plus aisé de percevoir leurs rapports extérieurs que leurs relations intimes et spirituelles; et les uns cachent le manque des autres. On croit souvent que la poésie limite la musique en s'unissant à elle, mais elle la rend aussi plus déterminée, plus perceptible - nous pourrions même dire plus humaine. ${ }^{23}$
\end{abstract}

soit au contraire on y voyait précisément le propre d'une musique " absolue » et " autonome ». C'est Rossini qu'Arthur Schopenhauer invoque en exemple dans sa «métaphysique de la musique " dans Le Monde comme Volonté et comme Représentation (supplément au livre troisième, chapitre XXXIX). Jean-François CANDONI (Penser la musique, p. 36) fait ainsi remarquer : « le terme [de musique absolue] désigne à l'origine une musique jugée purement décorative et divertissante (celle de Rossini ou de Meyerbeer) et n'exprimant aucune intention poétique. »

${ }^{22}$ KUNZE, Ludwig van Beethoven, p. 180 : « Andere halten die künstliche Ausbildung der Instrumentalmusik für das Verderbnis der Kunst - dies nicht ohne einen scheinbaren Grund, so fern die höchste Ausbildung eines Dinges zugleich den Keim seines Verfalles enthält. »

${ }^{23}$ Ibid., p. 173: "Ihre vollkommene, unsichtbare Macht übt die Tonkunst als Selbstherrscherin in der Instrumentalmusik; sie artet aber auch hier am leichtesten, bey dem, der die Geister der Töne nicht durch den Zauberstab des Genius zu beherrschen weiss, in ein 
De façon extrêmement surprenante, le lien de la musique avec la poésie lui permet de rester dans les limites de l'art. C'est une façon de reprendre la perspective des romantiques en la dirigeant contre leur promotion de la musique instrumentale. Si la musique doit éviter de dégénérer en artifice de composition sans expression, alors le texte n'est pas pour elle une barrière, mais un garde-fou. Ainsi, dans son unique opéra, Beethoven a su maîtriser ses tendances les plus anarchiques; la discipline plus exigeante de ce genre l'a contraint à freiner sa fougue et son imagination, qui débordent dans sa musique instrumentale. On sait que l'accouchement des œuvres vocales de Beethoven fut singulièrement douloureux : il a dû écrire trois versions de Fidelio sur presque dix ans, et il a consacré pas moins de cinq ans à l'écriture de la Missa solemnis. Or Wendt évalue ce difficile et lent travail de façon positive. Les efforts qu'a coûtés au génial Beethoven la production de ses œuvres vocales viennent de l'obligation de mettre sous le joug d'un texte une musique habituée à prendre ses aises. Et Wendt reproche par ailleurs au maître ses bizarreries, son excentricité, son «maniérisme ». Dans De l'état actuel de la musique, il va jusqu'à considérer que ses toutes dernières œuvres sont écrites par un sourd qui n'est plus capable de s'adresser à ceux qui peuvent et qui savent encore entendre ${ }^{24}$.

Wendt accorde aussi une attention particulièrement aiguë à l'emploi de la musique instrumentale dans son compte-rendu de Fidelio. Dans le fameux « air de la colère » de Pizarro, par exemple, il reconnaît que la musique ne se donne pas précisément pour tâche d'aider le baryton à prononcer le texte de façon intelligible. Mais ce défaut est compensé par l'expression violente de l'orchestre lui-même. Si le chanteur ne peut se faire entendre, c'est précisément que sa colère est trop forte et que l'orchestre se déchaîne à ce moment-là. Nous n'entendons plus des paroles, mais ce qu'elles expriment, directement, par la musique. De façon générale, Wendt pense que la musique instrumentale est arrivée à un stade de maturité qui lui permet d'être aussi expressive et déterminée, "caractéristique », que le texte qu'accompagne la musique vocale. L'évolution est considérable par rapport à Hoffmann. Celuici louait la musique instrumentale de Beethoven parce qu'elle nous ouvre un royaume ineffable et indicible. Wendt la félicite d'être parvenue à décrire les choses de façon aussi précise que les mots. C'est pour cette raison que non seulement il privilégie l'opéra dans sa triade classique, mais aussi que les progrès de la musique instrumentale y trouvent particulièrement leur place. L'opéra allemand laisse l'orchestre se développer, contrairement à l'opéra

künstlichen Spiel mit Tönen aus, deren äusserer Zusammenhang leichter wahrzunehmen ist, als die innere, geistige Verbindung; daher der Mangel dieser gewöhnlich hinter jenen verborgen wird. Wenn demnach die Musik durch Verbindung mit der Poesie beschränkt zu werden scheint, so wird sie auf der andern Seite auch bestimmter, vernehmlicher, ja wir möchten sagen menschlicher durch diese Verbindung. »

${ }^{24}$ WendT, Zustand, p. 41 : «Seine letzte große Messe aber, geschrieben in der einsamen Periode seines Lebens, wo der innere Sinn den verlorengegangenen äußern zu ersetzen strebte, ist überkünstelt ». (Sa dernière grande messe, qu'il a écrite dans la période solitaire de sa vie où le sens interne essayait de pallier l'absence du sens externe, est surchargée et artificielle.) 
italien, et c'est une discipline positive pour la musique instrumentale. Il l'empêche de suivre son chemin arbitraire, la guidant au contraire vers la vraie liberté.

Pour Robin Wallace ${ }^{25}$, ce qui sépare Wendt de Hoffmann est leur approche opposée de Beethoven : l'un cherche dans sa musique le développement puissant de son autonomie instrumentale, l'autre les limites salutaires de la musique vocale. Néanmoins, il faut ajouter que, selon Wendt, la musique instrumentale est parvenue dans l'opéra de Beethoven à une vérité dramatique qui n'invalide pas sa promotion par Hoffmann. Elle n'a plus pour vocation de nous plonger dans un monde métaphysique au-delà des mots, mais de nous peindre avec plus de précision que jamais les choses de ce monde. Pour Wendt, c'est avec les trois maîtres du classique que l'opéra a atteint son apogée. Et la musique y domine la poésie.

\begin{abstract}
Nous considèrerons tout d'abord l'opéra, parce que la musique vocale profane, qui est plus favorisée à l'ère moderne que la sacrée, a atteint là son sommet. Tout le monde sait (mais chacun s'en plaint) que la musique s'est si bien emparée de la scène qu'elle porte préjudice au drame, plus simple certes, mais laissant plus de place à la poésie. Il ne faut pas accuser seulement le manque de grands poètes tragiques ou comiques; la musique a fait tant de progrès et a si bien su élargir son domaine qu'elle supplante la poésie au sein même de l'opéra. Nous en sommes arrivés au point où les compositeurs, peu scrupuleux quant à la poésie propre du drame, font feu de tout bois, pourvu que l'emportement des passions représentées à la scène se prête à leur art et permette d'en montrer la puissance en mêlant à l'éclat visible de la scène celui de la musique. Ne nous étonnons pas que cela ait eu autant d'impact sur une foule qui veut se divertir les yeux et les oreilles, mais qui n'a qu'aversion pour la pensée libre. ${ }^{26}$
\end{abstract}

Wendt semble ici se plaindre de la prédominance de la musique sur la poésie comme de la victoire du divertissement sur l'art véritable. Mais dans Des grandes périodes des beaux-arts, au moment d'évoquer les apports de la musique italienne au début de l'ère baroque, il considère que dès sa naissance, l'opéra a fait prévaloir la musique sur le texte, ce qui le distingue du drame antique :

${ }^{25}$ RobinWallace, Beethoven's Critics. Aesthetic Dilemmas and Resolutions During the Composer's Lifetime, Cambridge, Cambridge University Press, 1986.

${ }^{26}$ WeNDT, Zustand, p. 22 : «Wir betrachten zuerst die Oper, weil in ihr die weltliche Gesangsmusik, welche in neuerer Zeit begünstiger ist, als die geistliche, ihren Gipfel erreichte. Allgemein bekannt und vielbeklagt ist die Erscheinung, daß dieselbe auf der Bühne allmählich eine solche Herrschaft gewonnen hat, daß sie auch dem einfachen, aber poetischerem Drama an allen Orten Eintrag thut. Der Grund davon ist nicht blos in einem Mangel an großen Tragödien und Lustspieldichtern zu suchen, sondern hängt wohl überhaupt mit der erfolgten Ausbildung und Verbreitung der Musik, und der dadurch entstandenen Herrschaft der Musik über die Poesie in der Oper selbst zusammen. Diese nehmlich ging so weit, daß die nach Poesie der Handlung wenig fragenden Componisten jeden rohen Stoff, welcher die Leidenschaften in einer lebhaften Bewegung darstellte, in ihren Kreis zogen, um an ihm die Macht ihrer Kunst zu zeigen und den Glanz der Musik mit dem sichtbaren Glanz, welchen ihnen die Bühne darbot, zu verschmelzen suchten. Kein Wunder, daß dies die schau- und hörlustige Menge, die dem freien Denken sich gern entzieht, gewaltig anzog. » 
Remarquons pour finir que c'est ici que les chemins du drame musical et du théâtre se séparent. Comme nous l'avons remarqué plus haut, cela est capital pour le drame moderne, autant que l'était pour le drame antique la stricte différenciation du tragique et du comique. Car si chez les Anciens la poésie dramatique réunissait tous les arts et que la musique se subordonnait, pour nous Modernes l'opéra a fait de la musique quelque chose d'essentiel, dont procède le drame, et qui rassemble tous les arts pour produire un effet commun. ${ }^{27}$

L'opéra apparaît ainsi comme le lieu où la musique parvient à son apogée sans être entravée par les autres arts, sur lesquels elle prédomine. Il est le sommet de la musique et même de l'art moderne en général, il émancipe totalement la musique alors même qu'il la relie aux autres arts. Il est donc bien le véritable modèle de la " période classique».

\section{Conclusion}

La plupart des commentateurs s'accordent à dire que l'esthétique de Wendt n'est jamais qu'une conséquence épigonale des Cours d'esthétique de Hegel, même si Wendt se fait gloire d'avoir écrit avec Des grandes périodes des beaux-arts la première histoire universelle de l'art, avant que Hotho publie les cours professés par le maître à Berlin, en 1835. Le plus souvent, l'esthétique hégélienne est présentée comme hostile à la musique instrumentale, ne disant pas un mot de celle de Beethoven, et voyant en elle un jeu vide et arbitraire des sons que ne guide aucune matière extérieure. Au contraire, la musique vocale et notamment l'opéra y sont présentés comme une possibilité pour la musique de rester dans les limites de l'art, grâce au contenu vivant du chant et de l'action. Cependant, Hegel réserve explicitement dans ses cours l'appellation de "période classique » à l'art de la Grèce antique, et au domaine de la sculpture et du drame. La musique y est présentée au contraire comme l'art romantique par excellence, dans la lignée de Tieck, Wackenroder et Hoffmann. On peut certes, comme Eggebrecht ${ }^{28}$, tenter avec brio et pénétration d'utiliser l'esthétique hégélienne pour lui faire dire ce qu'elle n'a jamais dit, à savoir que le "classique viennois » (die Wiener Klassik) peut être pensé grâce à la définition du classique par Hegel.

Mais c'est l'originalité de Wendt d'avoir dit en toutes lettres que la musique de Haydn, Mozart et Beethoven n'est pas romantique, mais classique, qu'elle représente non seulement des progrès propres à la musique

${ }^{27}$ WENDT, Hauptperioden, p. 274 : « Zuletzt bemerken wir noch, daß hier die Scheidung des musikalischen Dramas von dem Schauspiele beginnt, welche, wie oben bemerkt worden, eben so wichtig ist für das moderne Drama, als der strengere Unterschied des Tragischen und Komischen für das antike, indem, wenn dort die dramatische Poesie alle Künste vereinigte und die Musik sich unterordnete, hier die Musik, die nur der Oper wesentlich ist, ihr eignes Drama erzeugt und alle Künste zu einer gemeinsamen Wirkung verknüpft. »

${ }_{28}$ Hans Heinrich EGGEBRECHT, Versuch über die Wiener Klassik, Die Tanzszene in Mozarts Don Giovanni, Beihefte zum Archiv für Musikwissenschaft, gebundene Ausgabe, Stuttgart, Franz Steiner, 1972. 
instrumentale, mais aussi l'équilibre du chant et de l'instrumentation. Ce faisant, Wendt suggère que la notion de classique dans la musique implique de penser une musique capable de s'humaniser, de s'incarner en un personnage agissant réellement sous nos yeux. Bref, contre toute attente, la musique classique n'est pas la musique instrumentale du romantisme, mais la musique vocale de l'opéra. La musicologie allemande du XIX $\mathrm{e}^{\mathrm{e}}$ siècle ne cessera d'être aux prises avec ce débat opposant à la «musique absolue », qui ne signifie rien, une musique d'opéra ou même à programme, dont le sens est déterminé, aux dépens de son autonomie. 